\title{
Management of Mechanical Problems of Totally Implantable Venous Catheters
}

\author{
Halil Kara ${ }^{1}$ iD, Akif Enes Arikan² (D), Onur Dulgeroglu¹ (D), Cihan Uras² (iD \\ ${ }^{1}$ Acibadem Mehmet Ali Aydinlar University, Vocational School of Health Sciences, Istanbul, Turkey. \\ ${ }^{2}$ Acibadem Mehmet Ali Aydinlar University, School of Medicine, Department of General Surgery, Istanbul, Turkey.
}

Correspondence Author: Halil Kara

E-mail: halil.kara@acibadem.edu.tr

Received: $07.02 .2020 \quad$ Accepted: 11.03 .2020

\begin{abstract}
Objective: Although the most common complications of totally implantable venous catheters(TIVC) are infection and thrombosis, mechanical complications can also affect the treatment and cause catheter removal. This study aimed to investigate mechanical complications of TIVC and prevention methods.

Methods: Data of 983 procedures in 961patients who underwent TIVC implantation between 2010 and 2019 in AcibademMaslak, Bakirkoy, and Atakent Hospitals were retrospectively analyzed for mechanical complications.

Results: Mechanical complications were encountered in 33(3.3\%) cases: $12(1.2 \%)$ were detachment of TIVC, $8(0.8 \%)$ occlusions, $5(0.5 \%)$ pneumothorax, $1(0.1 \%)$ hemothorax, $1(0.1 \%)$ malposition, $1(0.1 \%)$ extravasation, $2(0.2 \%)$ TIVC rotation, $3(0.3 \%)$ skin necrosis and extrusions.

Conclusion: The catheter tip should be placed in distal superior vena cava, reservoir pocket must be sufficient in size, reservoir should be fixed to pectoral muscle or fascia at least two points with nonabsorbable sutures. Subcutaneous fatty tissue resection from reservoir pocket should be performed in obese patients. The nature of the withdrawn blood form Seldinger needle should be checked visually whether venous or not. Risk of pneumothorax and detachment can be reduced by inserting the catheter from $1 / 3$ outer part of the clavicle during percutaneous technique. While complication rate can be reduced by peroperative fluoroscopy use, control X-ray should be taken in symptomatic patients, not routinely. Malposition can be seen in the peroperative period and can usually be corrected by good manipulation. Percutaneous transcatheter retrieval in addition to surgery is the gold standard treatment for detachment of TIVC. The most important factors in preventing complications are surgical experience and good care.
\end{abstract}

Keywords: Totally implantable venous catheter, complication, detachment, malfunction, malposition.

\section{INTRODUCTION}

Totally implantable venous catheters (TIVC), which have become a part of oncologic therapies today, are being used especially for the administration of chemotherapy agents (13 ). They are more tolerable in daily life as there is no external unit like peripheral central catheters (2-7).

Although the most common complications are infection and thrombosis, mechanical complications such as catheter malposition, pneumothorax, catheter detachment, malfunction can also affect the treatment of the patient and cause catheter removal (7).

This study aimed to investigate the mechanical complications of TIVC and prevention methods.

This is a single-institution retrospective cohort study of oncologic patients who had TIVCs implanted by the same surgeon.

\section{METHODS}

Data of patients who underwent TIVC implantation between 2010 and 2019 in AcibademMaslak, Bakirkoy, and Atakent Hospitals were retrospectively analyzed. Non-oncologic indications were excluded.

TIVC implantations were performed in operating room under general anesthesia through subclavian vein(SV). In cases where the percutaneous technique failed, TIVCswereimplanted with open technique.

Catheter was inserted into the SVby Seldinger technique from $1 / 3$ outer part of the clavicle with the catheter tip directed to suprasternal notch and a sufficient pocket for reservoir was prepared two $\mathrm{cm}$ caudally. Under guidance of fluoroscopic examination, length of the catheter was adjusted so that tip of the catheter remained in superior vena cava(SVC) near right atrium(RA). Catheter is attached to reservoir and secured with lock mechanism. Reservoir was fixed on pectoral 
muscle fascia with 2/0 polypropylene sutures.After testing for catheter integrity, the system was filled with heparinized solution. Control chest X-ray was not performed if there were no complaints such asdyspnea, cough, arrhythmia or malfunction in postoperative period.

In open technique, cephalic vein was released from the incision made ondeltopectoral sulcus. Following the cut-down, the catheter was inserted into the vein and length of the catheter was adjusted similar as in percutaneous technique. The catheter was fixed to the vein by silk ligation. From the same incision, pocket for the reservoir was prepared and same procedures were applied as in percutaneous technique.

General TIVC care was performed every 40 days by experienced medical staff in chemotherapy units.

This study was approved by Ethical Review Board of Acibadem Mehmet Aydinlar University on 09 January 2020 with number of 2020/01.

\section{RESULTS}

Data of 983 procedures in 961 patients who underwent TIVC implantation were evaluated retrospectively. At the time of implantation, the median age was $48.6 \pm 12.3$ years, body mass index (BMI) was $26.04 \pm 4.79 \mathrm{~kg} / \mathrm{m}^{2}$. Primary malignancy was breast in 789 , gastrointestinal in 139 , and miscellaneous in 33 cases.

Total complication rate was $6 \%(n=60)$. Venous thrombosis ( $n=12,1.2 \%)$ and detachment of TIVC ( $n=12,1.2 \%)$ were the most common complications. Mechanical complications were encountered in $33(3.3 \%)$ cases: $12(1.2 \%)$ were detachment of TIVC, $8(0.8 \%)$ occlusions, $5(0.5 \%)$ pneumothorax, $1(0.1 \%)$ hemothorax, 1 (0.1\%) late malposition, $1(0.1 \%)$ extravasation, 2 (0.2\%) TIVC rotation, $3(0.3 \%)$ skin necrosis and port extrusions.

In patients with detachment of TIVC, reservoir and associated catheter was excised surgically, while embolizedfragment was removed percutaneously. Only in one patient, the catheter which was migrated to hearth wall could not be removed. The patient was followed-up with anticoagulant therapy and no complication was detected during 11 months' follow-up. TIVC explantation was required in patients with occlusion, extravasation, malposition and skin necrosis. Surgical correction was performed for port rotation. Thorax tube drainage was applied to three of five patients with symptomatic pneumothorax and to the patient with hemothorax. There was no mortality due to mechanical complications at mean $34 \pm 74$ months follow-up period.

\section{DISCUSSION}

TIVC improves quality of life and has lower infection risk than other catheter types, however, mechanical complications still can be encountered (1-3, 5-7). Complications rate decreases as surgical experience increases (8). However, they still increase hospitalizationduration, treatment cost, and morbidity and may cause delay in treatment (9).

\section{Tip of catheter}

There is no clear consensus on where the catheter tip should be; distal SVC, RA or atriocaval junction (6, 7, 10-12). The important thing is that the catheter tip should be in a high flow vein not to constantly contact vessel wall or not be in heart leading to arrhythmia. Placing the catheter in small caliber vessels increases endothelial damage, risk of thrombosis, vascular stenosis, and perforation (11). While Mudan et al (6) placed mid-atrial, considering that there is less risk of thrombosis, Machat et al (7) and Zhang et al (12) placed at distal SVC. During implantations, the patient should be well monitored forarrhythmias. In our clinic, the catheter tip is placed in SVC near RA, and no complications associated with its location were detected.

\section{Post-implantation X-ray}

In many clinics, peroperative fluoroscopy is used to control the position of catheter, but post-implantation X-ray control can be performed in centers without fluoroscopy (13). Kim et al. (4) reported that surgical experience, asepsis, and fluoroscopy use were effective in reducing surgical complications. X-ray control after fluoroscopy-guided implantation is controversial. Some centers advocate $X$-ray necessity due to pneumothorax risk $(6,7)$. In minimal pneumothorax, patient can be followed without thorax drainage, however, drainage should be considered in patients with respiratory complaints. Chest X-ray should be performed selectively according to patient's complaints, not routinely. Mudan et al. (6) routinely performed X-rays. Considering the pneumothorax rate of $1.2 \%(n=12)$ in their series, unnecessary X-ray was taken in 978 patients. Similarly, Velioğluet al. (14) routinely performed X-ray at postoperative period, however, considering pneumothorax rate of $0.8 \%$, $\mathrm{X}$-ray was unnecessarily taken in 2038 patients. However, in another study, routine X-ray use was found not cost-effective due to low complication risk (7). In our clinic, routine X-raywas not used if patient was asymptomatic.

\section{Subcutaneous thickness}

Two problems may arise related with patient's BMI and local subcutaneous thickness in the implantation area. The first problem is cosmetic dissatisfaction especially in patients with low subcutaneous tissue or in petite patients. In TIVC with smaller reservoir, catheter diameter also decreases, which is not preferreddue to increased occlusion risk. Therefore, it is necessary to inform patient before implantation for cosmetic results.

Contrary, in patients with high BMl or subcutaneous tissue thicker than $2 \mathrm{~cm}$, reservoir cannot be felt or reservoir remains too deep to place port access needle sufficiently. Because the reservoir is fixed to pectoral muscle fascia, tissue thickness over it gains importance. For this reason, subcutaneous tissue should be excised if needed. Fosh et al. (8) recommended to place port in subcutaneous pocket, rather than suturing to pectoral fascia and various authors suggested to prepare reservoir pocket superficially in fatty tissue in obese patients, however reservoir rotation risk increases, so we do not recommend $(3,6)$. 


\section{Arterial puncture}

Arterial puncture during implantation has been reported up to $11 \%(7,14,15)$. It can be distinguished by observing withdrawn blood from Seldinger needle by the color and pulsation of the blood. Generally, arterial puncture alone does not cause any complication, however, if dilatator or catheter is inserted complications such as hemothorax, arterial dissection, embolism, neck hematoma, pseudoaneurysm, a-v fistula, and thrombosis can be seen (7). Clinically unrecognized arterial puncture can be detected by fluoroscopy or subsequent chest X-ray (7). Risk of arterial puncturing can be reduced with USG guidance (7). Although USG is recommended for this purpose, we believe that USG is not crucial in experienced centers because of no arterial catheterization and associated complications seen in our clinic.

\section{Pneumothorax and Hemothorax}

These are the most feared and fatal complications. Pneumothorax is not expected with appropriate open surgical technique, but it has been reported in 1.5$6 \%$ with percutaneous technique (7). Zerati et al.(3) reported thatpneumothorax rate was $0.1 \%$ and USG is not necessary to reduce pneumothorax risk. In contrast, Mudan et al. (6) detected pneumothorax as $1.2 \%$ under USG guidance and recommended routine use. In this study, rate of pneumothorax was $0.5 \%(n=5)$ and hemothorax was $0.1 \%(n=1)$, so we conclude that USG guidance is not crucial in experienced centers. BMI of three patients with symptomaticpneumothorax was $19.92,19.48$ and $19.13 \mathrm{~kg} /$ $\mathrm{m}^{2}$ and $\mathrm{BMI}$ of the patient with hemothorax was $16.8 \mathrm{~kg} / \mathrm{m}^{2}$. Although statistical analysis cannot be performed due to low patient number, we think that more care should be taken in patients with low $\mathrm{BMI}$, considering the mean $\mathrm{BMI}$ was $26.04 \pm 4.79 \mathrm{~kg} / \mathrm{m}^{2}$ in this series.

Thorax drainage may not be necessary in all patients with pneumothorax. Li Ma et al. (10) observed pneumothorax in 9 (0.3\%) patients and drained two of them. Velioğlu et al. (14) detected pneumothorax in $16(0.8 \%)$ patients and 14 of these underwent thorax tube drainage. In this study, drainage was performed in three of five patients who had respiratory symptoms. Postoperative chest $\mathrm{X}$-ray is recommended in case of suspicion. In some centers, chest X-ray is recommended routinely, but in our center, we recommend only if the patient has symptoms.

\section{Skin erosion and extrusion}

It has been reported in literature at $0.7-5 \%(4,13,15)$. Incision site tension, repeated needle puncture, extravasation of chemotherapeutics may lead to skin erosion especially in lean patient (16). The reservoir pocket must be sufficient in size in order to reduce incision site tension (4). In cachectic patients, TIVC with a smaller reservoir can be used to reduce tension. Kim et al. (4) found $6(0.7 \%)$ erosions; TIVC was removed in two patients, debridement, irrigation and resuture in others. In this study, three patients had erosion, and two developed extrusions and TIVC removal was required in all patients. In series of Yanık et al. (15), decubitus developed in 121(3\%) patients, even though reservoirs were placed behind pectoral muscle in patients with very thin subcutaneous tissue.

\section{Malfunction}

General definition is inability to infuse fluids and/or aspiration of blood $(14,17)$. Considering that the primary purpose of TIVC is infusion, not aspiration, we believe that definition of malfunction should be only inability of infusion. It may be encountered in cases where catheter tipwas not be placed in correct position, kinking of catheter especially at entrance to vein, clot formation in tip of catheter, andport rotation (3). As catheter enters vein at right angles, it may increase risk of complications as it will reduce infusion flow rate and require more forceful injection. Fibrin sheath occurs around catheter within the first 24 hours after implantation (11). This fibrin sheath is usually fragmented but may cause occlusion if complete. In situations such as prolonged infusion time, injection of saline cannot be done, arm swelling, neck pain, and inability to puncture reservoir or extravasation, mechanical complications with fluoroscopic examination have been reported in $4.3 \%$ (7). In cases while fluids can be infused but blood cannot be withdrawn, once the catheter has been checked with X-ray and deep venous thrombosis has been excluded, catheters can still be used.

By minimizing use of catheter in procedures (i.e. blood drawing) other than infusion of chemotherapeutics, risk of catheter occlusion may be reduced (14). In cases of catheter occlusion with clot, fibrinolytic agent can be a choice.

In open technique, the catheter should not be angled to prevent malfunction. Additionally, extremely tight knotting should be avoided during ligation and fixation of catheter to vein.

\section{Port rotation}

Port rotation isa rare complication where reservoir is reversed and cannot be punctured with a needle $(10,15)$. Diagnosis can be made by physical examination or by X-ray. Yanık et al. (15) reported 4 (0.12\%) patients and Li Ma et al. (10) reported $3(0.10 \%)$ patients with port rotation. Treatment is revision of the reservoir. To prevent this complication, reservoir should be fixed to pectoral muscle or fascia at least from two points with nonabsorbable sutures. Port rotation was detected in 2 patients during years 2016-2017 when absorbable sutures were used as fixation sutures in this series. Diagnosis was made by physical examination and corrected by simple surgical intervention

\section{Malposition}

When upper extremity veins were used, orientation of catheter tip to a vessel except SVC or RA is called malposition (14). Risk of malposition has been reported $0.1-4.5 \%$ (1, $2,10,13,15$ ). Matiotti-Neto et al. (2) found no difference between open and percutaneous techniques. Malpositions 
usually occur at time of implantation, but may develop later in cases of increased intra-thoracic pressure (11).

Malposition of catheter inserted into the SV may occur in internal jugular vein (IJV) or vice versa, or in veins such as azygos/hemiazygos vein, internal mammary vein, or ipsilateral/contralateral SV $(7,11)$ (Fig. 1, 2). It can also form coil within vein or migrate into the subintimalzone (11). Such malpositions can be easily detected during fluoroscopy or postoperative chest X-ray. In case of suspicion, it is necessary to take two-way radiographies or evaluate with computed tomography (11).

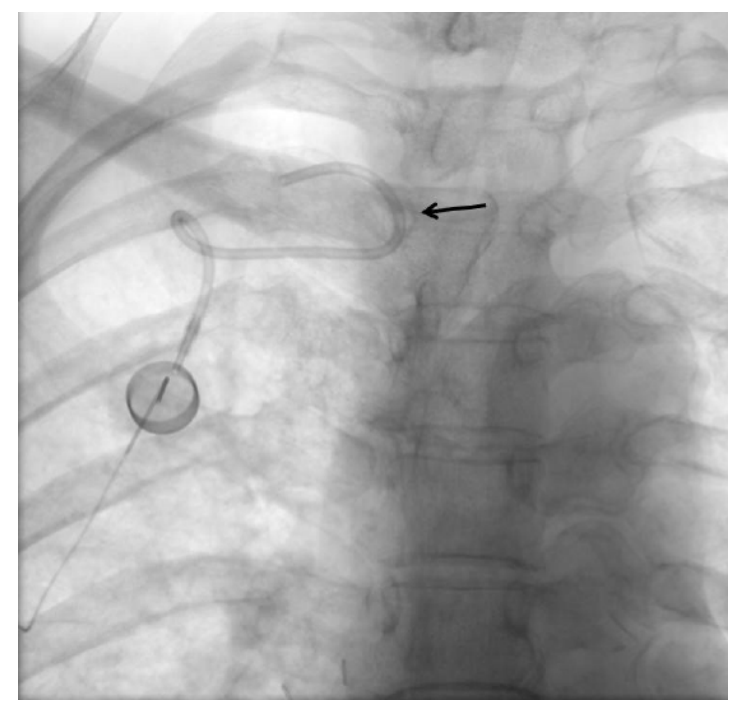

Figure 1. Malpositionof catheter to ipsilateral subclavian veinis indicated by black arrow

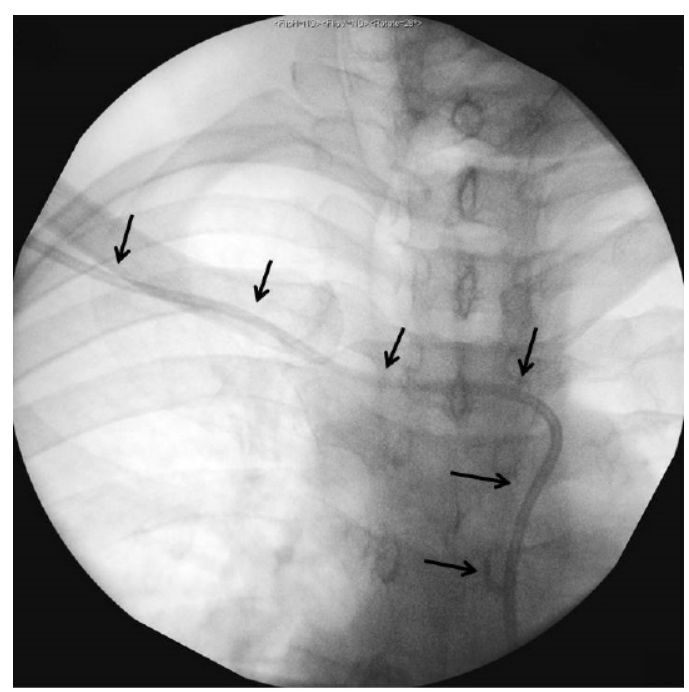

Figure 2. Malposition of catheter to hemiazygos vein. The catheter is indicated by black arrows

Venous thrombosis, erosion and perforation of the vessel wall, and catheter malfunction may be encountered due to malpositions.Various treatment methods can be tried (11). Catheter malposition can be corrected by forceful saline injection in small caliber catheters. If malposition is detected during implantation, inserting the guide wire into catheter and correcting malposition is an option.

Malposition of guide wire or catheter to contralateral SV during implantation is a condition we occasionally encounter (Fig. 3, 4). Correction of malpositions and orientation of catheter to SVC can be difficult. In such cases, we recommend to push forward the catheter rather than retracting and redirecting. Excess portion of catheter will be looped into the SVC,subsequently, when catheter is slowly retracted, tip will be placed in distal SVC by weight of the loop-shaped segment.

Malposition to IJVis rare in our clinic. To prevent this, patient is positioned to reverse Trendelenburg position while catheter is advanced.

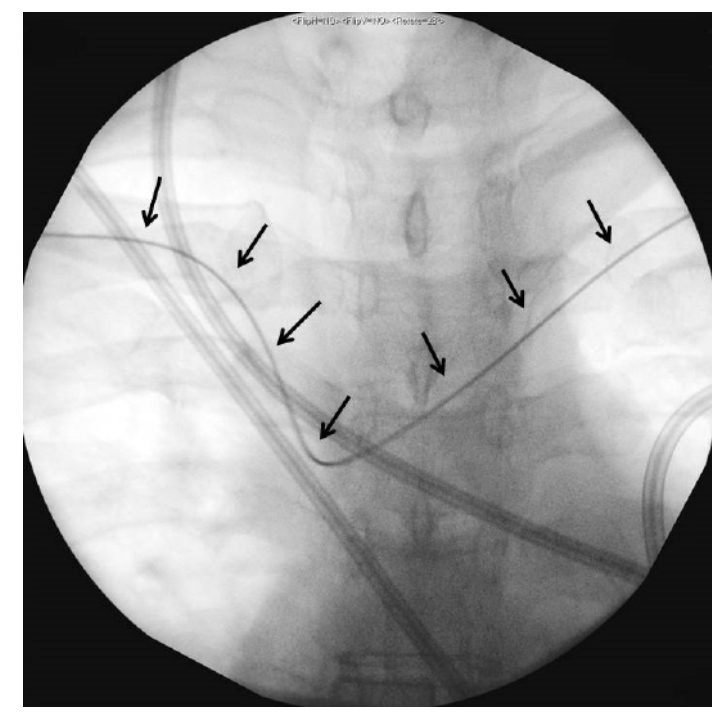

Figure 3. Malposition of guide wire to contralateral subclavian vein is indicated by black arrows

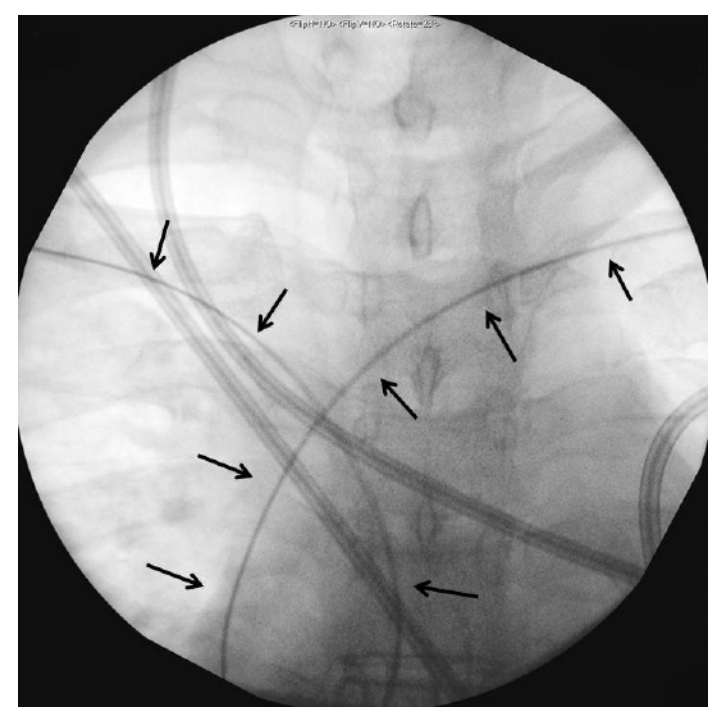

Figure 4. Loop formation during correction of the malposition to contralateral subclavian vein. Black arrows indicate the looped guide wire 
Malposition into heart during implantation may be seen. Therefore, arrhythmias up to $41 \%$ have been reported in the literature (7). Catheter length should be well adjusted, should not be advanced too much and site should be checked by fluoroscopy during implantation. The presence of catheter on left mediastinum or aortic arch should suggest intraarterial malposition (11). Extravascular malposition should be suspected in cases where the catheter does not follow the expected venous trace, and care should be taken in this respect during follow-up, especially in post-traumatic radiographs (11).

The diagnosis of late malposition can be made on follow-up radiographs. Although routine chest radiography is not recommended as late malposition is not common, X-rays taken in the follow-up of primary disease should be looked at in this respect or should be taken if in doubt. Leaving the catheter tip short at the SVC may cause late malposition. We think that if catheter tip is left distal SVC or atrial, late malposition will not occur due to length and weight of catheter.Late malposition was detected in one patients and TIVC was excised because of catheter dysfunction (Figure 1).

\section{Detachment}

It is a rare complication reported requiring treatment due to potential complications $(7,18)$. In this series, we detected in $12(1.2 \%)$ patients (Figure 5). It is usually seen after percutaneous SV catheterization, but it can also be seen when jugular vein is used or after open technique (19-22). Improper connection of catheter to reservoir, problems due to lock mechanism, incorrect manipulation, material fatigue, forced flushing, aggressive neck/extremity movements, trauma, orincreased intra-thoracic pressuremay lead todetachment of catheter. Pinch-off syndrome (POS) is the case where catheter is trapped and detached between 1st rib and clavicle $(5,9$, $11,23,24)$.Surov et al. (21) reported POS (40.9\%) as the most common cause of detachment, there was no etiologic factor in $19.1 \%$ of cases. In contrast, Chang et al.(24) reported fracture at the connection between reservoir and catheter (77 of 92 patient-84\%) as the most common cause. It may be due to technical or production error (25). POS (66\%) was the most common cause in this series.

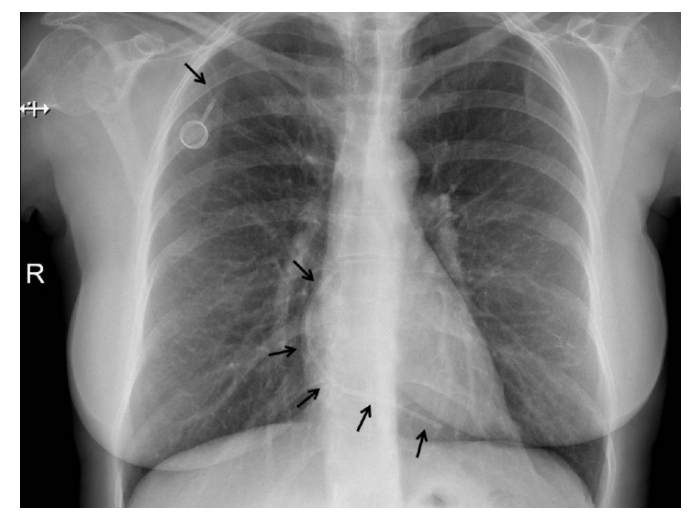

Figure 5. Detachment and embolization of catheter to heart. The point of detachment and embolized catheter fragment is indicated by black arrows
Most of the patients were asymptomatic and diagnosis was made by routine chest X-ray $(3,5)$. However, catheter malfunction, arrhythmia, pulmonary symptoms, septic syndromes, severe pain, inability to draw blood, extravasation, edema around the catheter and reservoir, and pain can also be seen $(9,21,24,26)$.

To prevent POS, insertion of catheter from lateral $1 / 3$ of the clavicle is recommended $(9,23,27)$. Two-stage treatment therapy is recommended (23). Percutaneous transcatheter retrieval of embolized fragment in addition to surgical removal of reservoir and attached catheter is the gold standard $(5,9,21,23,24,26,28)$. If percutaneous removal is impossible, it can be removed by thoracotomy or followed by anticoagulant treatment for a long period $(5,21,24,29)$.

\section{Experience and TIVC care}

Experience of healthcare personnel is important in preventing complications. TIVC implantation following guidelines and with good care, complication rate can be reduced (6). Ertel et al. (30) reported that rate of complications was related to clinician implanting TIVC more than technique. Fosh et al. (8) draw attention to learning curve and report that complication rate is reduced with surgical experience. Particular care should be taken during port access needle entry toavoid accidental damage to catheter. Additionally, attention should be paid to infusion pressure range of implanted catheter.

It is recommended to wait for healing ofincision before use of TIVC, and flush catheter with heparinized solution after each use or at most 4-6 weeks $(1,5)$. However, Zhang et al. (12) stated that port can be used on implanted day. In our clinic, TIVCs are used by experienced nurses in the next day following implantation and we did not detect any complications associated with early use.

\section{CONCLUSION}

Diagnosis and treatment of mechanical complications of TIVCs are important to prevent delay in the treatment of the patient.The catheter tip should be placed in SVC near RA, the reservoir pocket must be sufficient in size, reservoir should be fixed to pectoral muscle or fascia at least from two points with nonabsorbable sutures. Subcutaneous fatty tissue resection should be performed in obese patients. The nature of the withdrawn blood form Seldinger needle should be checked visually whether venous or not. Risk of pneumothorax and detachment can be reduced by inserting the catheter from $1 / 3$ outer part of the clavicle in percutaneous technique. While complication rate can be reduced by peroperative fluoroscopy use, control X-ray should be taken in symptomatic patients, not routinely. Malposition can be seen in the peroperative period and can usually be corrected by good manipulation. Percutaneous transcatheter retrieval in addition to surgery is the gold standard treatment for detachment of TIVC. The most important factors in preventing complications are surgical experience and good care.

Funding: No funding was received. 
Conflicts of Interest: There is no conflict of interest regarding the publication of this article.

Informed consent: Informed consent was not needed due to being a retrospective study.

\section{REFERENCES}

[1] Kock HJ, Pietsch M, Krause U, Wilke H, Eigler FW. Implantable vascular access systems: experience in 1500 patients with totally implanted central venous port systems. World J Surg 1998; 22: 12-16.

[2] Matiotti-Neto M, Eskander MF, Tabatabaie O, Kasumova G, Bliss LA, Ng SC, Tawa NE, Jr., Murphy B, Critchlow JF, Tseng JF. Percutaneous versus Cut-Down Technique for Indwelling Port Placement. Am Surg 2017; 83: 1336-1342.

[3] Zerati AE, Wolosker N, de Luccia N, Puech-Leao P. Totally implantable venous catheters: history, implanttaion technique and complications. J Vasc Bras 2017; 16: 128-139.

[4] Kim DH, Ryu DY, Jung HJ, Lee SS. Evaluation of complications of totally implantable central venous port system insertion. Exp Ther Med 2019; 17: 2013-2018.

[5] Intagliata E, Basile F, Vecchio R. Totally implantable catheter migration and its percutaneous retrieval: case report and review of the literature. G Chir 2017; 37: 211-215.

[6] Mudan S, Giakoustidis A, Morrison D, losifidou S, Raobaikady R, Neofytou K, Stebbing J. 1000 Port-A-Cath (R) placements by subclavian vein approach: single surgeon experience. World J Surg 2015; 39: 328-334.

[7] Machat S, Eisenhuber E, Pfarl G, Stubler J, Koelblinger C, Zacherl J, Schima W. Complications of central venous port systems: a pictorial review. Insights Imaging 2019; 10: 86-97.

[8] Fosh B, Canepa M, Eaton M. Long-term venous access insertion: 'the learning curve'. ANZ J Surg 2016; 86: 1038-1041.

[9] Ilhan BM, Sormaz IC, Turkay R. Pinch-Off Syndrome, a Rare Complication of Totally Implantable Venous Access Device Implantation: A Case Series and Literature Review. Korean J Thorac Cardiovasc Surg 2018; 51: 333-337.

[10] Ma LI, Liu Y, Wang J, Chang Y, Yu L, Geng C. Totally implantable venous access port systems and associated complications: A single-institution retrospective analysis of 2,996 breast cancer patients. Mol Clin Oncol 2016; 4: 456-460.

[11] Kidney DD, Nguyen DT, Deutsch LS. Radiologic evaluation and management of malfunctioning long-term central vein catheters. AJR Am J Roentgenol 1998; 171: 1251-1257.

[12] Zhang P, Du J, Fan C, Mo X, Dong J, Fan Z, Zhao Q. Utility of totally implantable venous access ports in patients with breast cancer. Breast J 2019; 26: 333-334.

[13] An H, Ryu CG, Jung EJ, Kang HJ, Paik JH, Yang JH, Hwang DY. Insertion of totally implantable central venous access devices by surgeons. Ann Coloproctol 2015; 31: 63-67.

[14] Velioğlu Y, Yüksel A, Sınmaz E. Complications and management strategies of totally implantable venous access port insertion through percutaneous subclavian vein. Turk Gogus Kalp Damar Cerrahisi Derg 2019; 27: 499-507.

[15] Yanik F, Karamustafaoglu Y, Karataş A, Yoruk Y. Experience in totally implantable venous port catheter: Analysis of 3,000 patients in 12 years. Turk Gogus Kalp Damar Cerrahisi Derg 2018; 26: 422-428.

[16] Zerati AE, Figueredo TR, de Moraes RD, da Cruz AM, da MottaLeal Filho JM, Freire MP, Wolosker N, de Luccia N. Risk factors for infectious and noninfectious complications of totally implantable venous catheters in cancer patients. J Vasc Surg Venous Lymphat Disord 2016; 4: 200-205.

[17] Goossens GA, Stas M, Jerome M, Moons P. Systematic review: malfunction of totally implantable venous access devices in cancer patients. Support Care Cancer 2011; 19: 883-898.

[18] Turner DD, Sommers SC. Accidental passage of a polyethylene catheter from cubital vein to right atrium; report of a fatal case. N Engl J Med 1954; 251: 744-745.

[19] Pignataro BS, Nishinari K, Wolosker N, Bomfim GA. Fracture and migration into the coronary sinus of a totally implantable catheter introduced via the right internal jugular vein. BMJ Case Rep 2014; 2014:1-3.

[20] Mirza B, Vanek VW, Kupensky DT. Pinch-off syndrome: case report and collective review of the literature. Am Surg 2004; 70: 635-644.

[21] Surov A, Wienke A, Carter JM, Stoevesandt D, Behrmann C, Spielmann RP, Werdan K, Buerke M. Intravascular embolization of venous catheter-causes, clinical signs, and management: a systematic review. JPEN J Parenter Enteral Nutr 2009; 33: 677685.

[22] Saijo F, Mutoh M, Tokumine J, Yoshinobu O, Hama H, Namima $\mathrm{T}$, Shibahara M, Tokumura H. Late fracture of Groshong ports: A report of the three cases. J Vasc Access 2019; 20: 563-566.

[23] Motta Leal Filho JM, Carnevale FC, Nasser F, Santos AC, Sousa Junior Wde O, Zurstrassen CE, Affonso BB, Moreira AM. Endovascular techniques and procedures, methods for removal of intravascular foreign bodies. Rev Bras Cir Cardiovasc 2010; 25: 202-208.

[24] Cheng CC, Tsai TN, Yang CC, Han CL. Percutaneous retrieval of dislodged totally implantable central venous access system in 92 cases: experience in a single hospital. Eur J Radiol 2009; 69: 346-350.

[25] da Motta Leal Filho JM, Carnevale FC, Cerri GG. Subclavian vein an unusual access for the removal of intravascular foreign bodies. Ann Vasc Surg 2010; 24: 826 e821-824.

[26] Gowda MR, Gowda RM, Khan IA, Punukollu G, Chand SP, Bixon $R$, Reede DL. Positional ventricular tachycardia from a fractured mediport catheter with right ventricular migration-a case report. Angiology 2004; 55: 557-560.

[27] Aitken DR, Minton JP. The "pinch-off sign": a warning of impending problems with permanent subclavian catheters. Am J Surg 1984; 148: 633-636.

[28] Carroll MI, Ahanchi SS, Kim JH, Panneton JM. Endovascular foreign body retrieval. J Vasc Surg 2013; 57: 459-463.

[29] Tazzioli G, Gargaglia E, Vecchioni I, Papi S, Di Blasio P, Rossi R. Retained embolized fragment of totally implantable central venous catheter in right ventricle: it is really necessary to remove? J Vasc Access 2015; 16: 431-433.

[30] Ertel AE, McHenry ZD, Venkatesan VK, Hanseman DJ, Wima K, Hoehn RS, Shah SA, Abbott DE. Surgeon, not technique, defines outcomes after central venous port insertion. J Surg Res 2017; 209: 220-226. 